\title{
More than looking good: effect and effectiveness of physical activity, functional body image and quality of life in people sustaining a spinal cord lesion
}

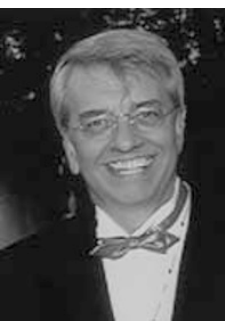

JJ Wyndaele, Editor

Antwerp University Hospital, Antwerp, Belgium

E-mail: spinalcord@uza.be

Dear Spinal Cord reader,

This March issue is very rich in content.

Harvey et al. have methodologically reviewed the quality of evaluations of the effectiveness of various types of physical intervention, including fitness and strength training, gait training, hand therapy, stretch, acupuncture and hand splinting. Some trials reported a between-group mean difference with a clearly important treatment effect on at least one outcome measure supporting the use of fitness, strength and gait training, as well as acupuncture.

Jannink et al. assessed the effect of exercise therapy on motor control and functional ability of the upper extremity in patients with cervical spinal cord injury (SCI) through an extensive systematic literature search. Of the small number of included studies, most reported positive effects of exercise therapy. Future studies should be more specific in describing the characteristics of the exercises in order to verify that they are in accordance with the current standards for training and motor relearning.

Ashe et al. determined physiatrists' opinions and current practice patterns for bone-health management after spinal cord injury and found more support for pharmacological treatments than for rehabilitation modalities.

Bassett and Ginis examined the body image-leisure time physical activity relationship among men with SCI. In individuals who reported a negative effect of body image on quality of life (QOL), those who engaged in leisure time physical activity were less satisfied with their physical function than those who did not. For those who did not perceive their body image to negatively affect their QOL, there was generally no difference in functional satisfaction according to whether individuals performed leisure time physical activity or not. Body dissatisfaction may motivate some men living with SCI to engage in leisure time physical activity, but this would influence functional body image only when a negative effect on QOL is perceived.

Allen et al. showed that the motor function of children after paediatric SCI depends on neurological level and completeness of injury, among other factors. They also showed that functional independence measure motor scores can improve with intervention even years after the injury.

In this issue, we continue with the International Spinal Cord Injury Data Set series. Krogh et al. made a high standard basic and extended data set, both of which are presented here.

A special contribution is from Dajue Wang, who has written a compact literature review to provide basic knowledge on reticular formation for clinicians. Besides a review of the anatomical structure and related areas, clinical considerations are made on multi-system damage, muscle contraction, upper urinary tract, sexual behaviour, skin tropic, pain, sleep apnoea, cross-system damages, spinal cord repair and comprehensive management.

These are just some examples of the scientific content. There are many more high-quality scientific contributions, including original articles, case reports and book reviews. There is also a letter to the editor.

I am convinced you will find all of them important and useful. 